القدرة على الاتحاد لتراكيب وراثية من القطن الابلند من خلال الجيل الثابي لتهجين تبادلي بين سبعة أصناف

$$
\text { خالد خليل الجبوري } 1
$$

النبات وعالياً لبقية الصفات. اما التحسين الوراثي المثوقع من

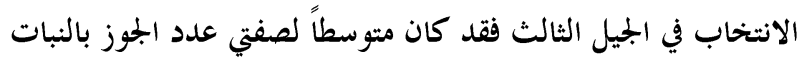

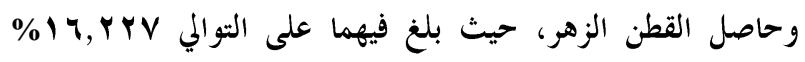

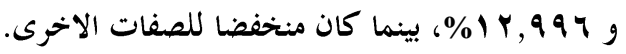

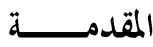

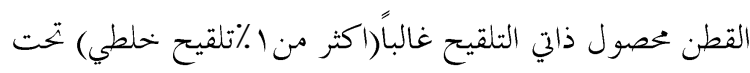

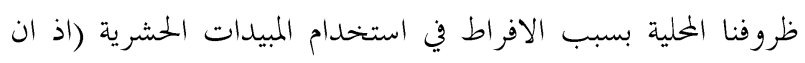

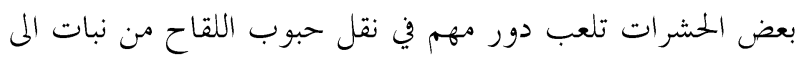

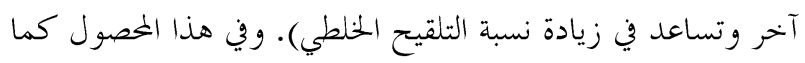

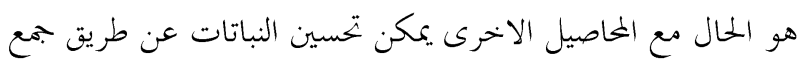

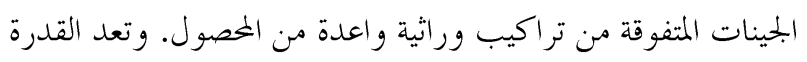

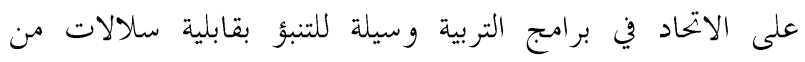
المصول الاتحادية للصفات المختلفة ومن ثم اختيار المفضلة منها.

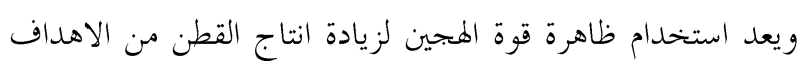

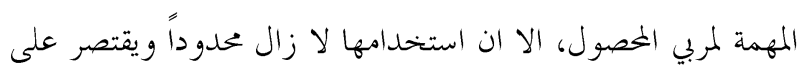

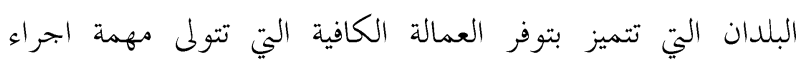

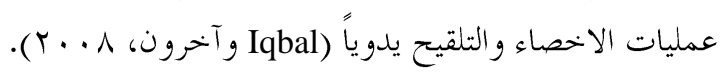

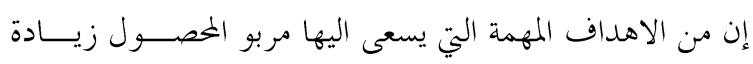

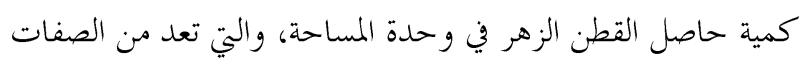

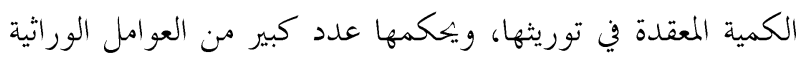

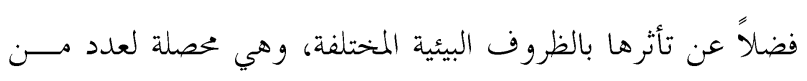

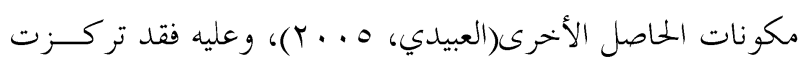

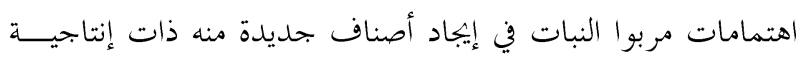

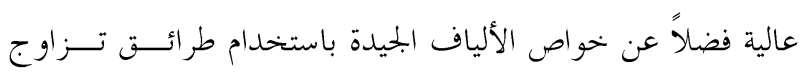

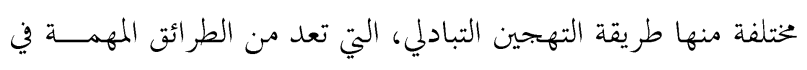

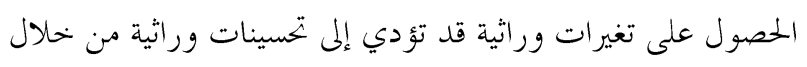

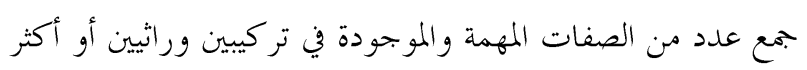

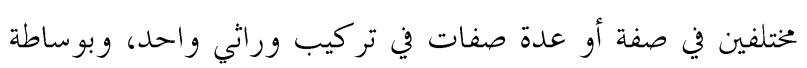

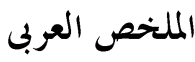

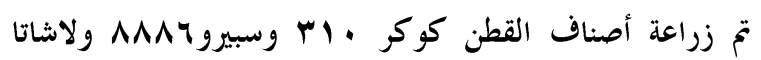

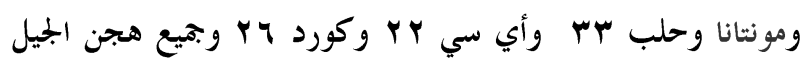

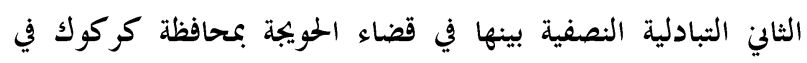

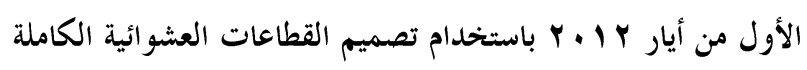
بثلاثة مكررات، لدراسة تأثير ات القدرتين العامة والحاصة على على الاتحاد التاد

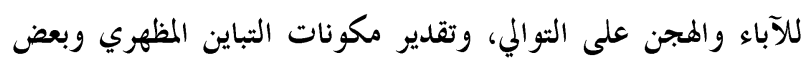

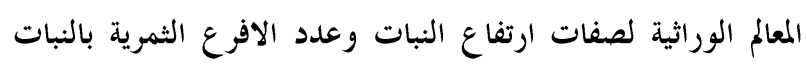

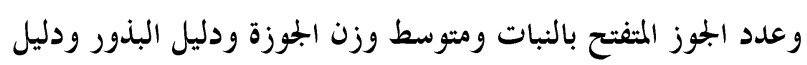

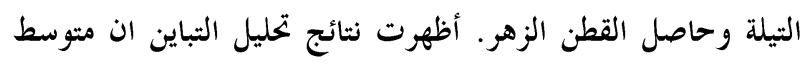

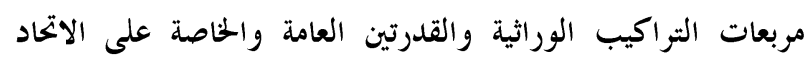

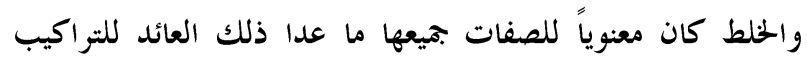

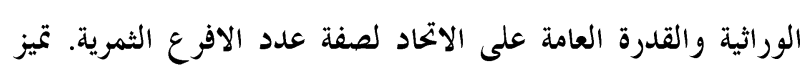

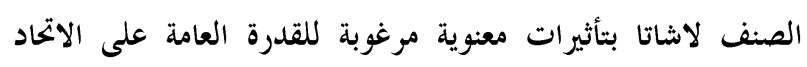

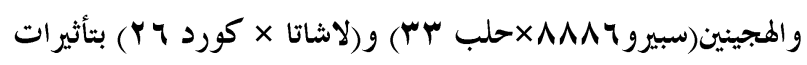

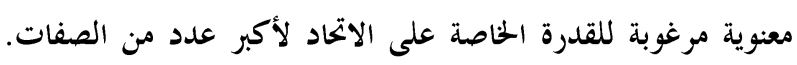

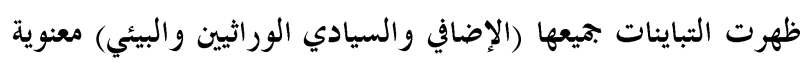

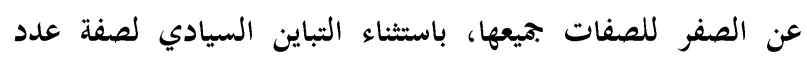

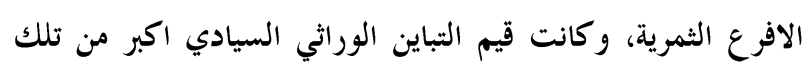

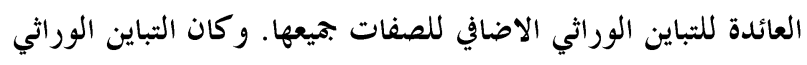

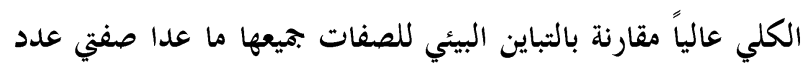

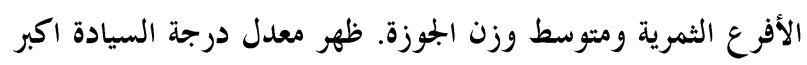

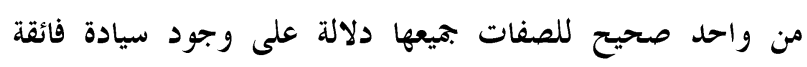

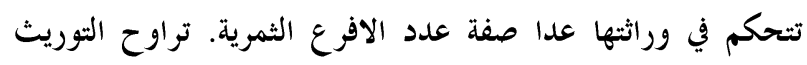

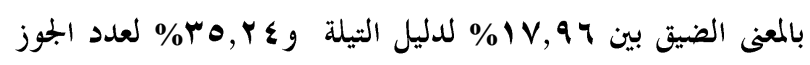

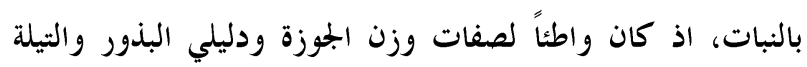

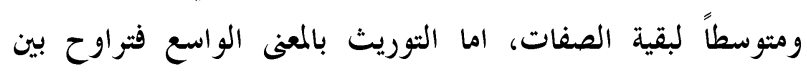

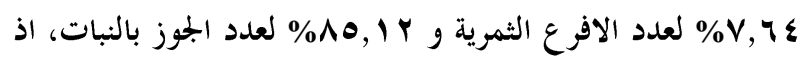

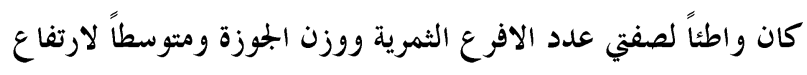

أقسم الخاصيل الحقلية، كلية الزراعة، جامعة كركوك، العراق

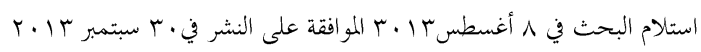




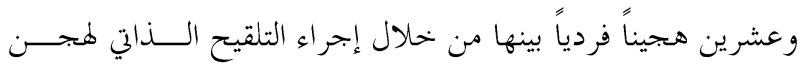

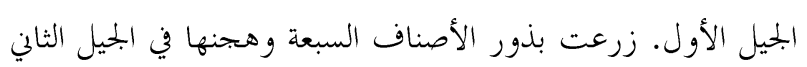

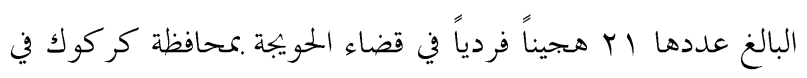

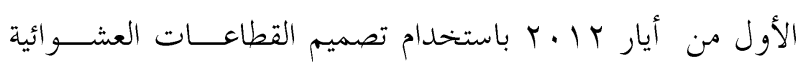

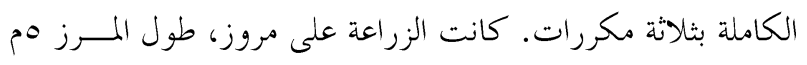

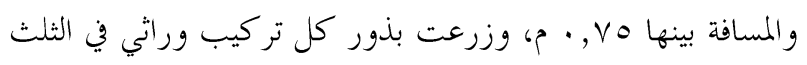

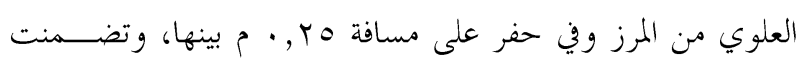

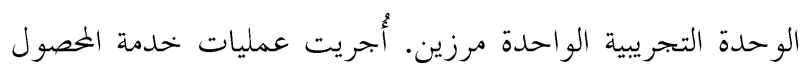

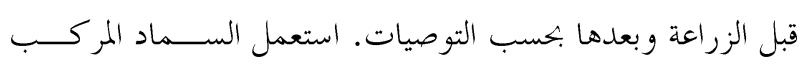
(NPK)

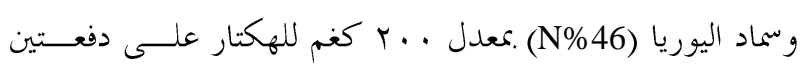

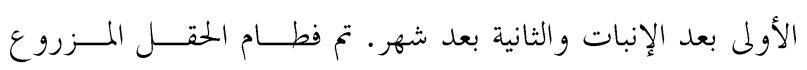
بالمصول في بداية أيلول وجنيت النباتات الفردية (عشرة نباتـات

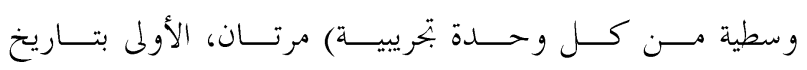

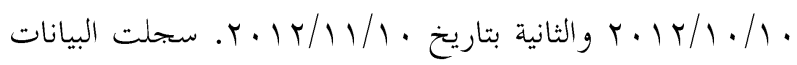

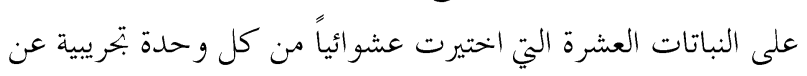

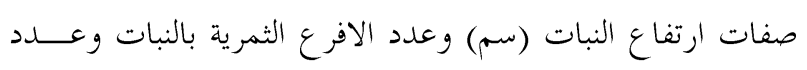

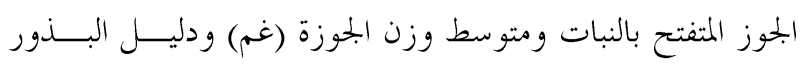

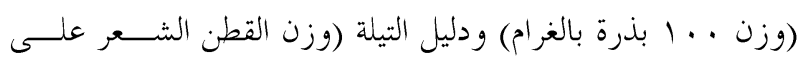

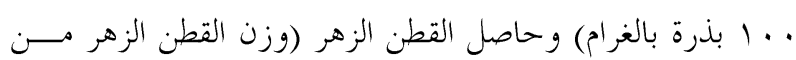
الجنيتين لكل نبات بالغرام).

حللت بيانات التراكيب الوراثية (الأصناف وهجنها في الجيـل لحل

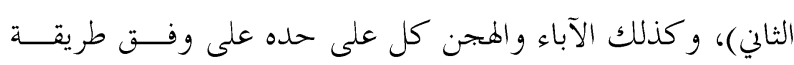

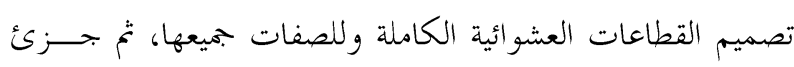

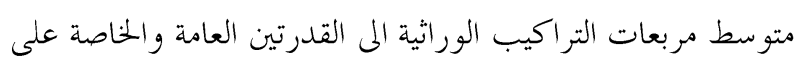

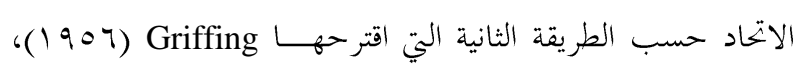

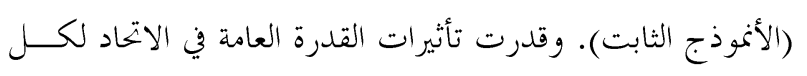

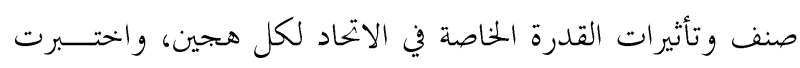

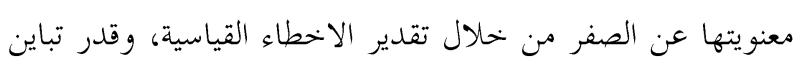
تأثير القدرتين العامة والخاصة لكل صنف. تم تقدير التدهور بالتربية

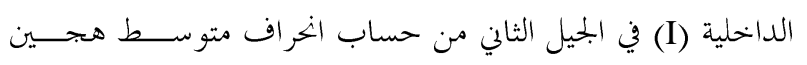

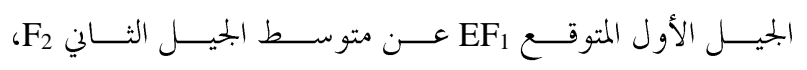
$\mathrm{I}=\mathrm{EF}_{1}$ - ،(199V ،Hassang 1990 ،Shaheeng Gomma)

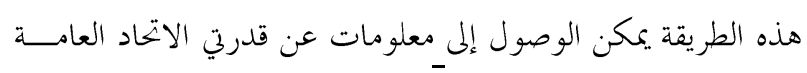

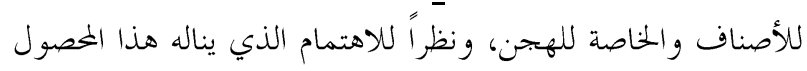

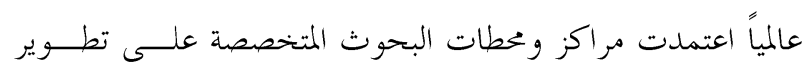
أصناف متميزة منه باستمرار ولاسيما في الدول التي تنتجهُ بكميات كبيرة، من حلال تبني الدراسات التي كتم بتقدير تباينات وتأثيرات

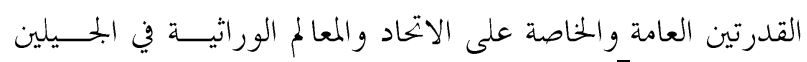

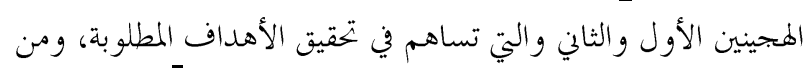

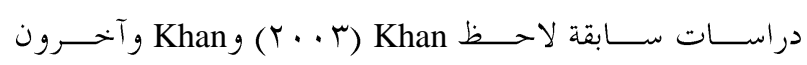

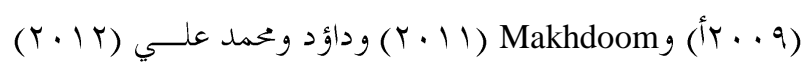

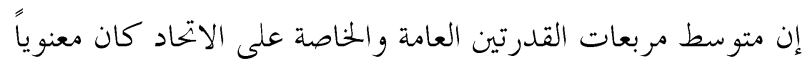

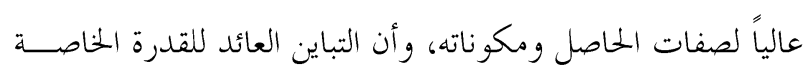

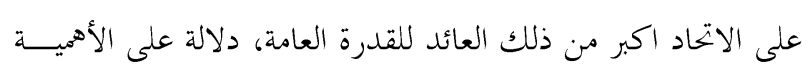

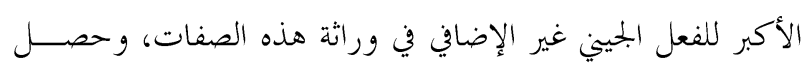
Iqbal

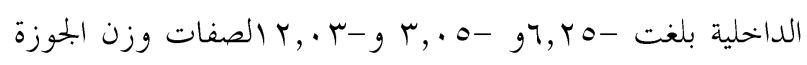

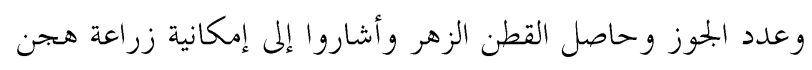

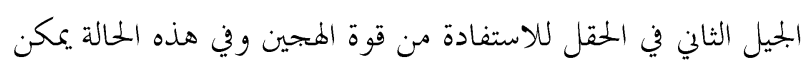

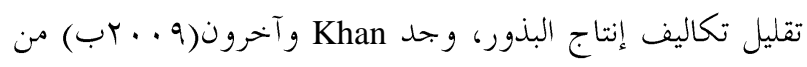
هجين تبادلي الختلافات معنوية بين متوسطات التراكيب الوراثية في

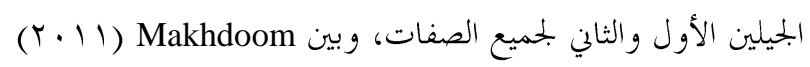
أن الآباء ذوات القدرة العامة على الاتحاد (عالي x و واطئ) و و(واطئ

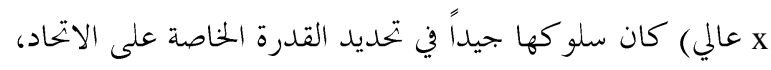
كدف الدراسة الحمالية الى تقويم أصناف من القطــنـ وهجنــــها

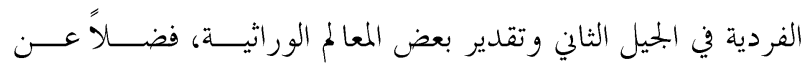

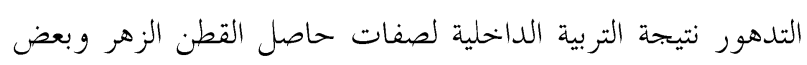
مكوناته من الصفات الاخرى.

\section{مو اد البحث وطرائقه}

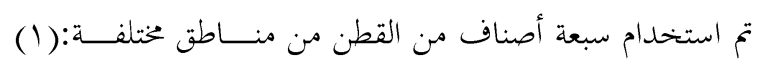

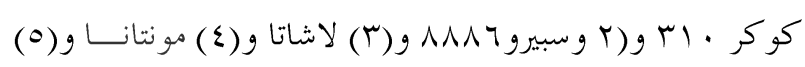

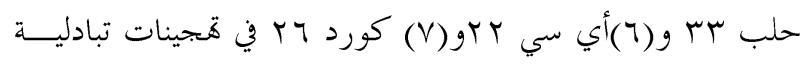

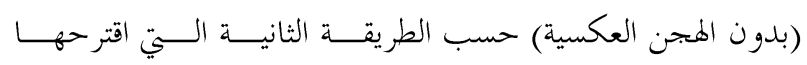

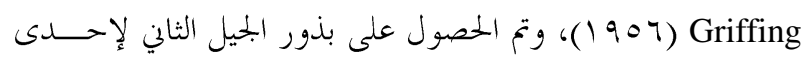




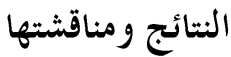

يظهر في الجدول(1) نتائج تحليل التباين للتراكيب الوراثية (الآباء

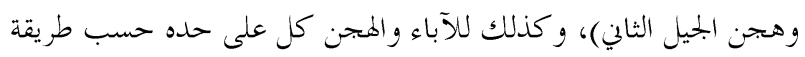

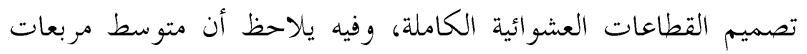

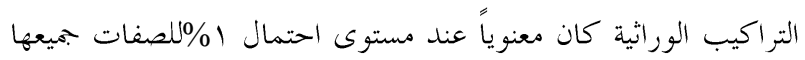

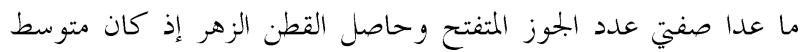

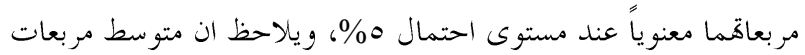

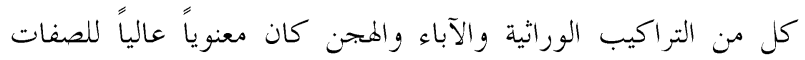

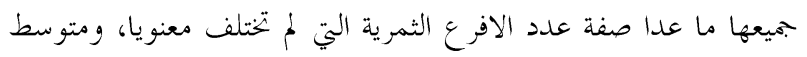

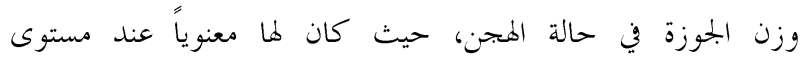

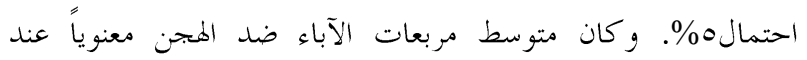
مستوى احتمال ا \% لصفات عدد الافرع الثمرية وعدد الجوز المتفتح بالنبات ودليلي البذور والتيلة، وعند مستوى احتماله\% لبقية الصفات.

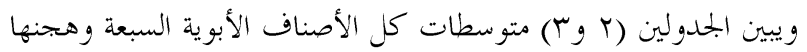

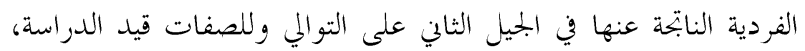

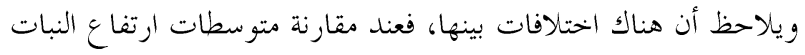

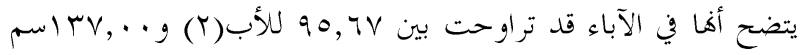

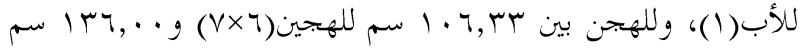

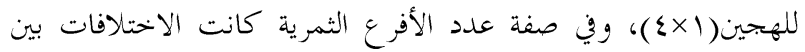

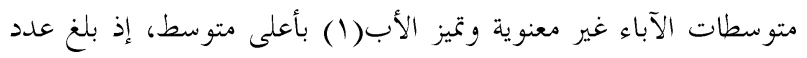

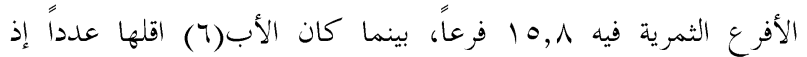

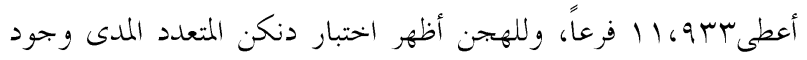

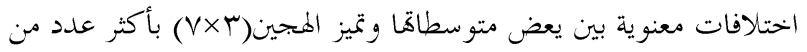

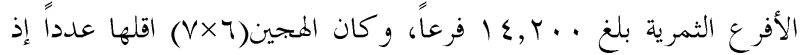

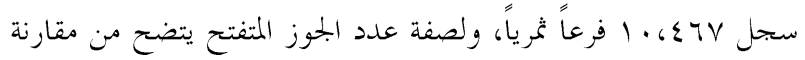

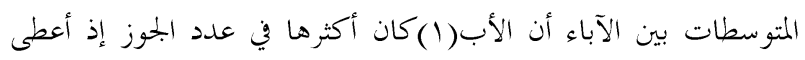
Y Yroo

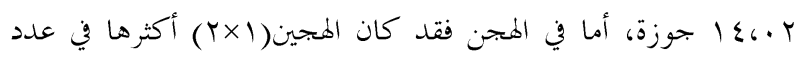

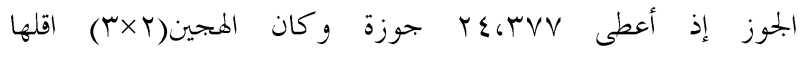
وأعطى بrه، • ا جوزة. تراوحت قيم متوسط وزن الجوزة في الآباء بين

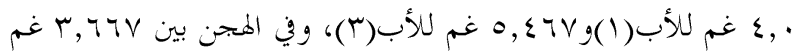

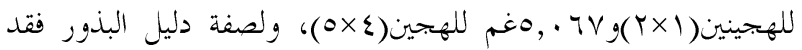

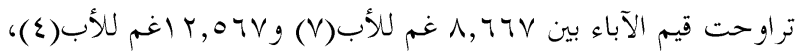

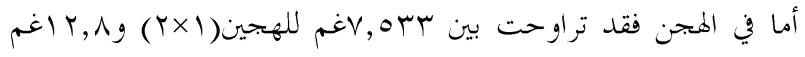

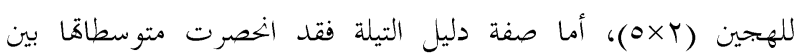

F2 علماً أن المتوسط المتوقع لهجين الجيل الأول قــــر باسـتخدام المعادلة الــي أوضـــا 1/2P1-1/2P

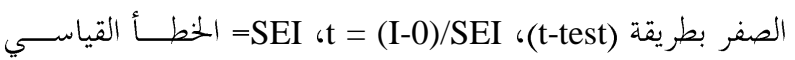

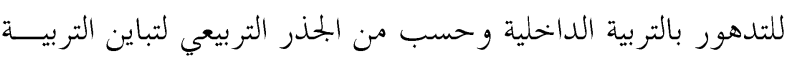

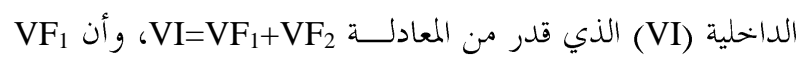
تعني تباين متوسط الجيلين الأول والثاني على التوالي. قدر التباين المظهري مَ من بحموع ع التباين الوراثي الاضــافي

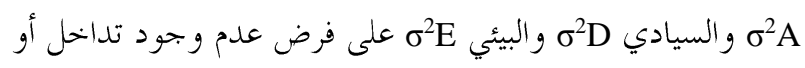

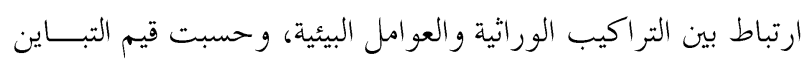

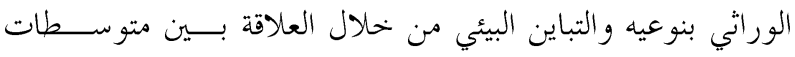

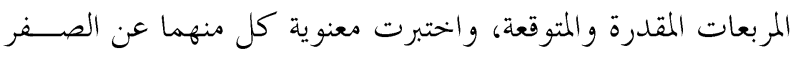
بالطريقة التي أوضحها Kempthorne (190V ) من خلال حساب

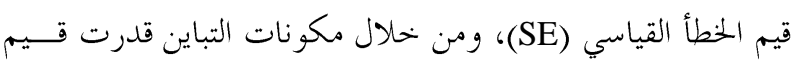

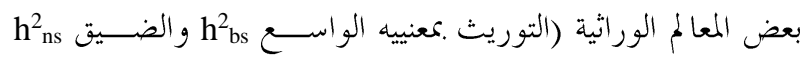

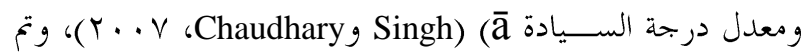
اعتماد حدود التوريث بالمعنى الواسع حسب علي (9999 (199)، (أقل

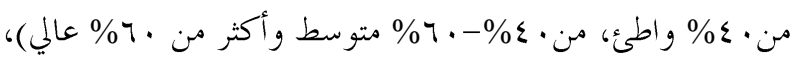

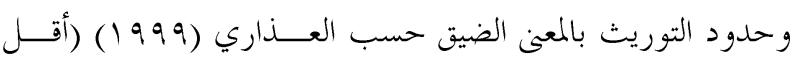

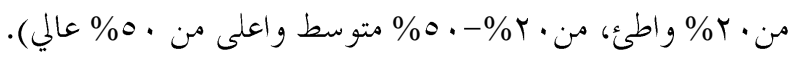
قدر التحسين الوراثي المتوقع في الجيل الثالث (GA) لكل صفة

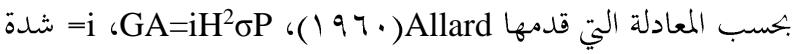

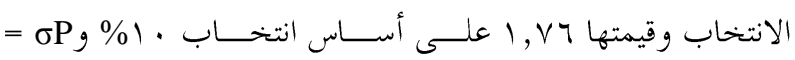

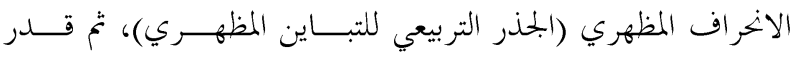

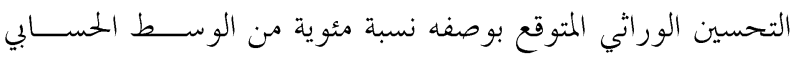

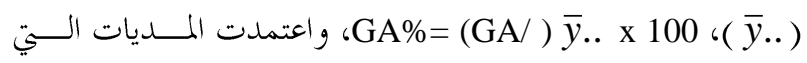

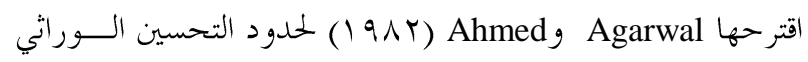

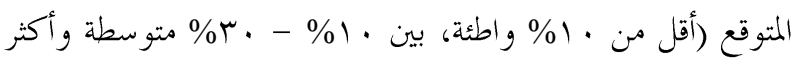

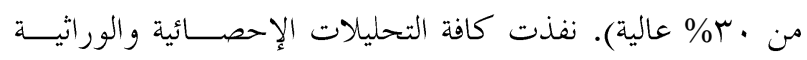

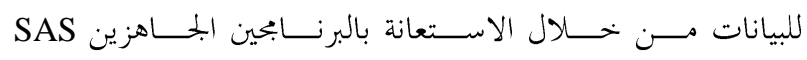
Microsoft Office Excel g (Statistical Analysis System) 2003 


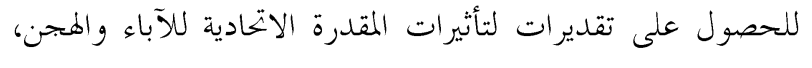

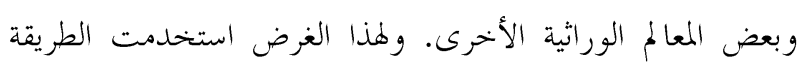

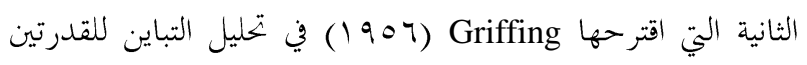
العامة والخاصة على الاتحاد، للأصناف الأبوية وهجن الجيل الثاني

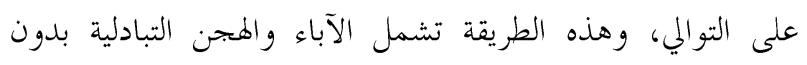

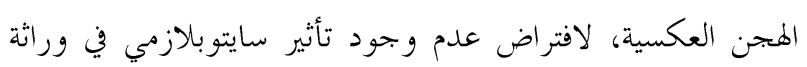

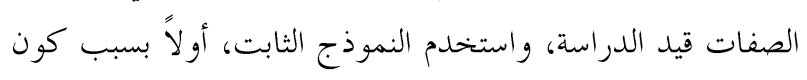

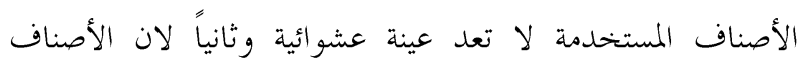
وهجنها زرعت في موقع واحد، وهنا تعد تأثيرات العوامل البيئية

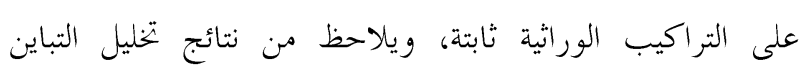

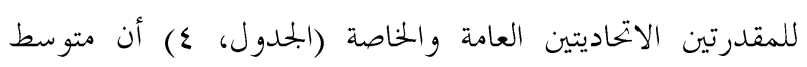

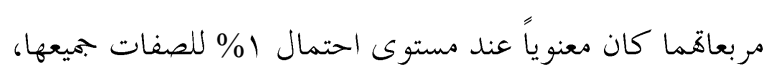

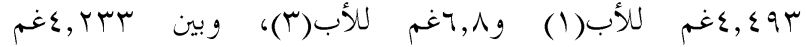

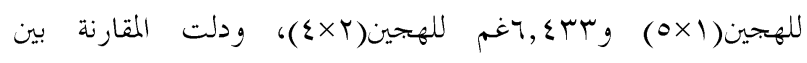

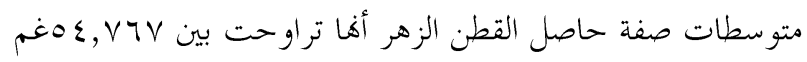

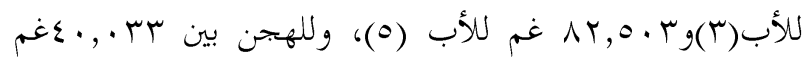

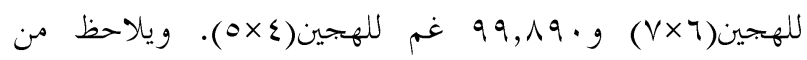

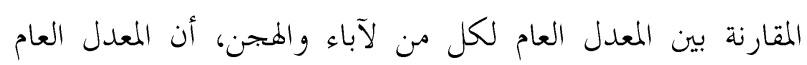

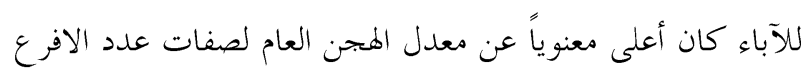
الثمرية بالنبات ومتوسط وزن الجوزة ودليلي البذور والتيلة دلالة

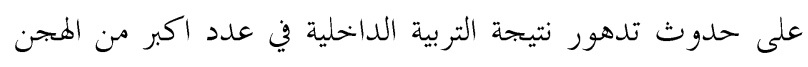
لهذه الصفات، بينما كان العكس بالنسبة للصفات الثلاث الاخرى. إن وجود الاختلافات الوراثية بين الآباء وهجن الجيل الثاني النابتة بلنة

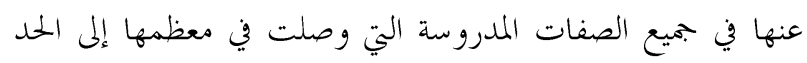
المعنوي بتعل من الضروري الاستمرار في التحليل الإحصائي الوراثي

جدول ا ـ نتائج تحليل التباين للتراكيب الوراثية بموجب تصميم القطاعات العشوائية الكاملة للصفات المدروسة

\begin{tabular}{|c|c|c|c|c|c|c|c|c|}
\hline \multicolumn{7}{|c|}{ الصفات } & \multirow[b]{2}{*}{ درجات } & \multirow[b]{2}{*}{ مصادر التباين } \\
\hline الزهر بالنبات القطن & دليل التيلة & البذليل & وزن الجموزة & عالنبات الجوز & علثد الافرع & النبات (سم) ارتفاع & & \\
\hline 0991,00 & $r, V \backslash \wedge$ & YO TV $T$ & $9,7 \leqslant 7$ & $Y \circ Y, V Y$ & $170, \Sigma$. & $r q \wedge \varepsilon, 7 \varepsilon$ & r & القطاعات \\
\hline ****Tฯ Tr, TV & **** $1, \vee \succ$ & *** & ${ }^{*} \cdot, \Sigma \vee T$ & $* * 0 \cdot, r$ & $\varepsilon, I V V$ & **** $\quad \backslash \leqslant \wedge, q$ & TV & التر اكيبالور اثية \\
\hline 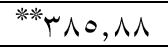 & ****, Y, I & **** ч, r。 & ***.,,$\wedge \varepsilon$ & 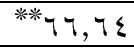 & $0, \vee 0 \varepsilon$ & **** $\circ \wedge \cdot, \wedge$ & 7 & الآباء/أ \\
\hline ***VVY,AY & $* * * 1,09$ & ***, YY & $*, r \circ q$ & ****ध一 $\{, \backslash \wedge$ & $r, 190$ & **** 107,9 & $r$. & الهجن/هـ \\
\hline ש & ***** $1, \vee q$ & ***** $\uparrow \wedge, 9$ & *.,, $0 \leqslant r$ & ****, 0 Y & **** & $* q 0,0 \wedge r$ & 1 & أ ضد هـ \\
\hline $0 \xi, O V Y$ & $\cdot, r \leq r$ & $\cdot, 0 \leqslant \mu$ & $\cdot, r \cdot \Lambda$ & $r, \ldots r$ & $r, \diamond \wedge 0$ & $0 Y, Y 0$ & $0 \varepsilon$ & خطأ التراكيب \\
\hline$\sum \Lambda, T Y \mu$ & $\cdot$, YVY & $\cdot, \varepsilon \varepsilon r$ & $\cdot, Y \backslash \leqslant$ & $r, \cdot v \wedge$ & $7, \ldots 0$ & $\{1, .11$ & IT & خطأ الآباء \\
\hline 01, YYo & $\cdot, r V Y$ &., $0 Y V$ & $\cdot, Y M$ & $r, \ldots \varepsilon$ & $r, r_{0}$ & Tr,T & $\varepsilon$. & خطأ المجن \\
\hline
\end{tabular}

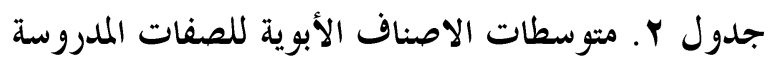

\begin{tabular}{|c|c|c|c|c|c|c|c|}
\hline \multicolumn{7}{|c|}{ الصفات } & \multirow[b]{2}{*}{ الوراثية } \\
\hline 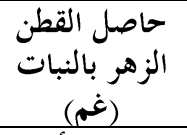 & دليل التيلة & دليل البذور & وزن الجوزة & عدالنبات الجوز & الثمرية بالنبات & 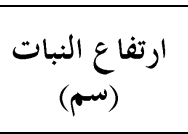 & \\
\hline$i \wedge \cdot, 0 \mid \mathrm{V}$ & $د \varepsilon, \varepsilon q \pi$ & ب, 9,0 V V & $2 \varepsilon, \ldots$ & I YY,O\&. & $i 10,1 \ldots$ & $i$ i rv,... & 1 \\
\hline سאו, דם ج & س & $\{11, \varepsilon \ldots$ & س r, & $ج \mid \varepsilon, \leqslant T V$ & $\{\mid r, r \ldots$ & T TV...90 & $r$ \\
\hline T O\&,VTV & $i_{\uparrow, \wedge \ldots}$ & س & i $0, \Sigma T \mathrm{~V}$ & $21 \cdot, 7 \ldots$ & $\{\mid \varepsilon, Y \backslash V$ & . & $r$ \\
\hline أب $v \vee q 1 V$ & $i 7,7 \leqslant r$ & $i \mid r, 07 \mathrm{~V}$ & ב & أب & $i \mid r, \varepsilon \ldots$ & ب 1. & $\varepsilon$ \\
\hline$i \wedge r, 0 . r$ & . . . . أب & II Y,YTV & س r, & ب । & $i, r, \wedge$. & $ب 11 \wedge, 7 \mathrm{~V}$ & 0 \\
\hline . & & $i 11, \Sigma v$. & . . . أب, أب & $\tau \mid \varepsilon, \cdot r$. & I I I, & $ب 11 \wedge, 7 \mathrm{~V}$ & 7 \\
\hline . . . & T & ر ب & . . . & 2 & $\{, r, V T r$ & $i|r|, r$. & $\mathrm{V}$ \\
\hline$v \cdot, I V$ & $0, V Y$ & $11,1 r$ & $\varepsilon, 70$ & 17,1 . & $1 \%, 17$ & IIV,ry & المعدل \\
\hline
\end{tabular}


جدولr. متوسطات هجن الجيل الثاني للصفات المدروسة

\begin{tabular}{|c|c|c|c|c|c|c|c|}
\hline \multicolumn{7}{|c|}{ الصفات } & \multirow[b]{2}{*}{ الوراثية } \\
\hline $\begin{array}{c}\text { الزهر بالنبات القطن } \\
\text { الزم) }\end{array}$ & دليل التيلة & دليل البذور (غم) & وزن الجموزة & عدالنبات الجوز & الثرية بالنبات & $\begin{array}{c}\text { ارتفاع النبات } \\
\text { (سم) }\end{array}$ & \\
\hline & jg,ror & ط r v,or & T,TYTV & $i r \varepsilon, r V V$ & VT., rا أب & 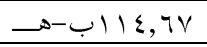 & $r \times 1$ \\
\hline $200,7 \ldots$ & $\rightarrow-I_{0}, V \sim r$ & . & أب & س & أب & $\rightarrow-119,7 \mathrm{~V}$ & $r \times 1$ \\
\hline$ج+\lambda 1,0 \leqslant V$ & r r T, هـ وز & r r r r, & T & Tr,rov & . . . & i i r & $\varepsilon \times 1$ \\
\hline & j $\varepsilon, Y \mu r$ & $b \tau^{\wedge}, \cdot T V$ & & س & سrا,11 أب & سr, זrוأب ج & $0 \times 1$ \\
\hline sor, . TV & سM, & $z j g \wedge, q \ldots$ & & jglr,V VV & سبر,11 أب & سr, r أب ج & $7 \times 1$ \\
\hline$\rightarrow 0 ., r V V$ & D & سr.،.1اج-و & 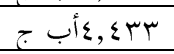 & jgIr,V.. & VIV & ه-اب-ه, & $v \times 1$ \\
\hline M O OY, & $2-i\rceil, 1 \ldots$ & & & j1.,人 & ب.,.7V & & $r \times Y$ \\
\hline$r$ rV,TOV & i & $9-T^{9, \wedge T V}$ & 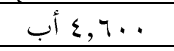 & ه- $19,1 \mu v$ & . .... & . & $\varepsilon \times Y$ \\
\hline ج & سس, س أب & $i, r, \wedge \ldots$ & . . . . & $\rightarrow 1 \wedge, \ldots$ & V & . & OXY \\
\hline ج & 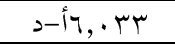 & 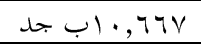 & r r & $\rightarrow 1 \wedge, r T V$ & VITr,r أب & Tro,TV Iأب T & $7 \times Y$ \\
\hline ج ب & $j \varepsilon, r \varepsilon$. & $\tau^{-} \rightarrow q, r \ldots$ & . & & . . & $\rightarrow-11 \varepsilon, T V$ & $V \times Y$ \\
\hline TVY,TTV & سMr,0ب-ز & j-د9,77V & . & $\rightarrow \mid V, r r r$ & . . . ا أب & $\rightarrow-I|r|, T V$ & $\varepsilon \times r$ \\
\hline س M & $2-10, \wedge 1$. & rس,q,q ד-פ & س سד, & سrו, rا وز & & . & oxr \\
\hline$د T \cdot r \cdot V$ & سMه, Iأ-هـ & س & سسر,ع أب & $g \mid \varepsilon, Y \neg V$ & سبو,11 أب & أب 1 ( $9,7 V$ & $7 \times r$ \\
\hline$\tau \vee V \varepsilon, \cdot \wedge V$ & . & س سو,.. اب جد & $\varepsilon, 17 V$ & $\rightarrow$ & $i \mid \varepsilon, r \ldots$ & $\rightarrow-ب \vee V, T V$ & $V \times r$ \\
\hline $199, \wedge 9$. & $j-7_{0,1}^{0, .}$ & $b_{\tau} \wedge, \ldots$ & $10, .7 \mathrm{~V}$ & $\rightarrow 1 \wedge, r q V$ & . . . 11, أب & س س الب-هـ & $0 \times \varepsilon$ \\
\hline ج & ز & rسM, זגן ל & س ., سع ب ج & $\rightarrow-r ., \Sigma \wedge v$ & سسו, זו أب & Vاب, • rاب-هـ & $7 \times \varepsilon$ \\
\hline أب & 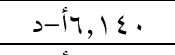 & . & 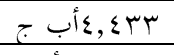 & $\rightarrow-4, q, \wedge r$. & & $د$ I I I T TV & $V \times \varepsilon$ \\
\hline T بV,... & $g-I_{0,0 Y .}$ & سM & أب & $\rightarrow I V, q Y r$ & 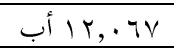 & ه-بر . TV & $7 \times 0$ \\
\hline أب & r & . . 11, أب & & & VIr,rו أب & . & $v \times 0$ \\
\hline$\rightarrow \varepsilon \cdot, \cdot \mu$ & $j-s 0, \cdot r V$ & $z^{-} \rightarrow 9,17 V$ & سسז,ییأب جـ & jg11,r... & ب $1 ., \Sigma 7 V$ & $\rightarrow 1 \cdot 7, \pi r$ & $V \times y$ \\
\hline$V Y, V$ & $0, \mu_{q}$ & $q, \vee V$ & $\varepsilon, \varepsilon 7$ & IV,or & $|Y, Y|$ & $119, \wedge r$ & المعدل \\
\hline
\end{tabular}

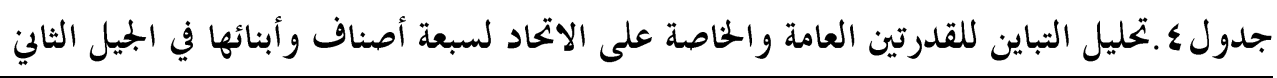
الصفات

\begin{tabular}{|c|c|c|c|c|c|c|c|c|}
\hline الزهر بالنبات & دليل التيلة & دليل البذور & وزن الجوزة & عدالنبات الجوز & عدالثمرية الافرع & ارتفاع النبات & درجات & مصادر \\
\hline 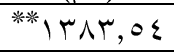 & ***, Y,VY & 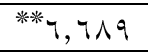 & ***.,,$\wedge \wedge$ & ****1.96V r & $r, 1 \mu$ & *** $\{\nearrow \varepsilon, \wedge q$ & 7 & GCA \\
\hline$* * * \leqslant \circ \uparrow, \vee$. & *** $1, \leqslant 9$ & $*^{* * *}$ Y, Y०r & $*, \mu, \xi$ & ***r, & * $\varepsilon, \varepsilon \vee 0$ & 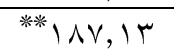 & YI & SCA \\
\hline $0 \xi, O V Y$ & $\cdot, \Gamma \leqslant r$ & $\cdot, 0 \leqslant \pi$ & $\cdot, r \cdot \Lambda$ & $r, \ldots r$ & r,०^० & $O T, Y \circ Y$ & $0 \leqslant$ & الخطأ \\
\hline$\cdot, r \neg V$ & . &., 119 &., $01 \%$ & $\cdot, r q r$ & . & $\cdot, r \leqslant V$ & ØGC & Ø SCA \\
\hline
\end{tabular}

و Turgut

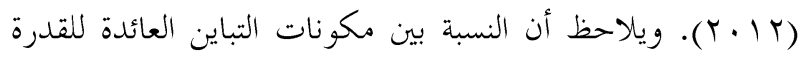

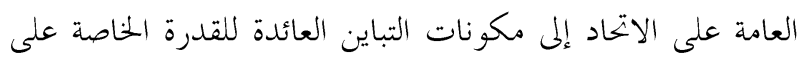

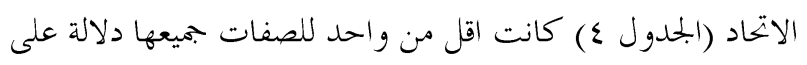
أن التأثيرات الجمينية غير الإضافية اكبر من التأثيرات الجحينية الإضافية

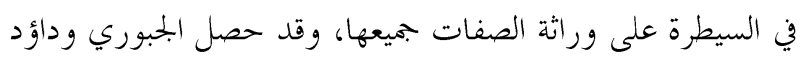

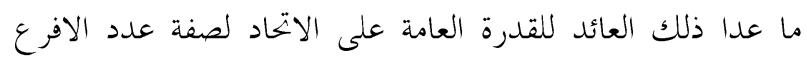
الثمرية (لم يصل اللى الحد المعنوي)، وللقدرة الخناصة على الاتحاد

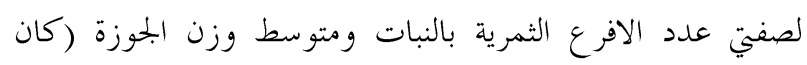
معنوياً عند مستوى احتمال ه\%)، ويستنتج من ذلك أن التأثيرات الجينية الإضافية وغير الإضافية تسيطر على وراثة جميع الصفات

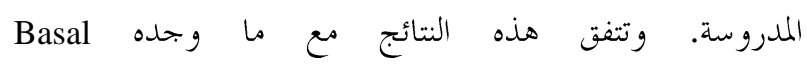


النبات أن الهجن (

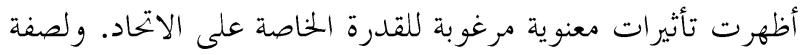

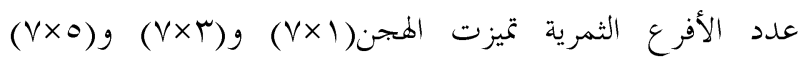
بتأثيرات معنوية مرغوبة للقدرة الخاصة على الاتحاد، وفي صفة عداد

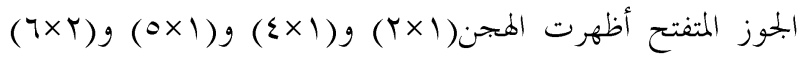
و

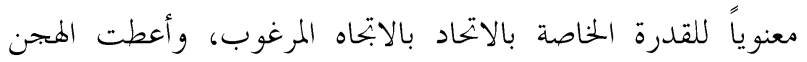

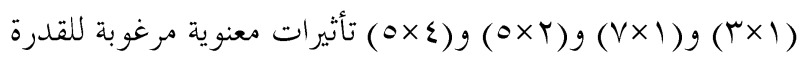

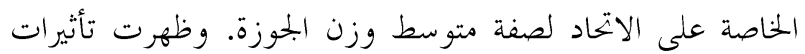
معنوية مرغوبة في سبعة هجن لكل من دليل البذور ودليل التيلة وحاصل القطن الزهر بلغت اعلاها للصفات الثلاث على التوالي

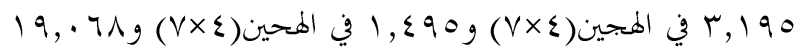
في الهجين(VXY). ويمكن من خلال هذه النتائج الاستفادة من الهجن

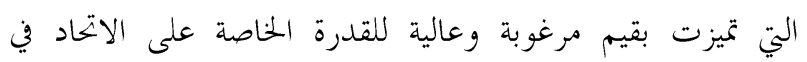

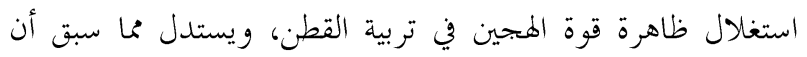

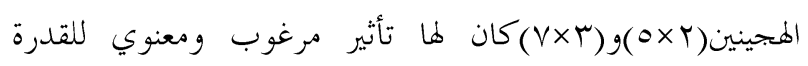

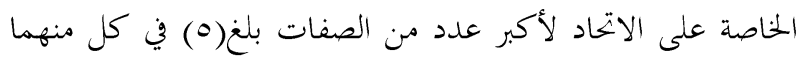

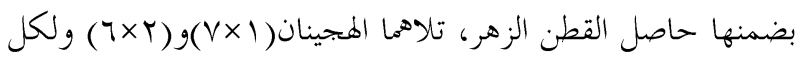

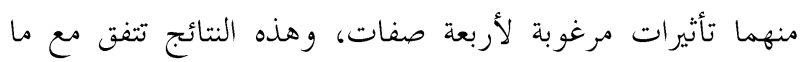

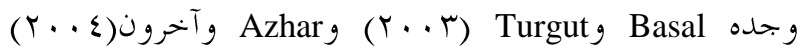

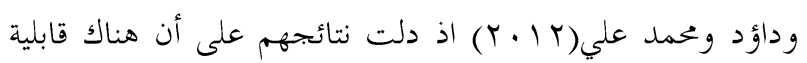
اتحاد خاصة معنوية ومرغوبة لبعض الهجن التي تضمنتها تجاربهم ولعدد من الصفات.

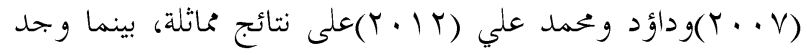
Iqbal

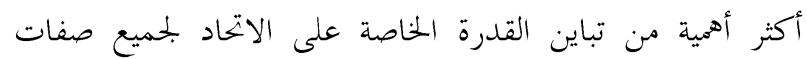

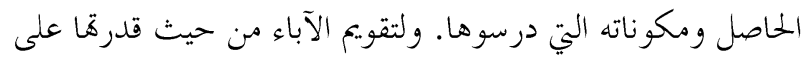

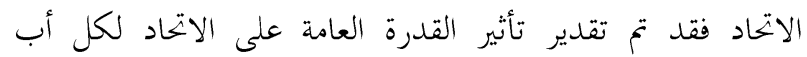
(الجدول، 0)، ويلاحظ فيه أن الأب (1) يتحد معنوياً في الابتحاه

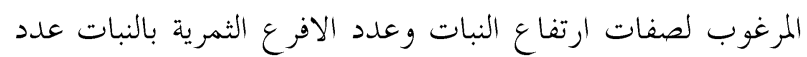

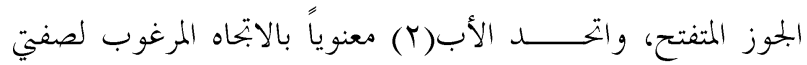

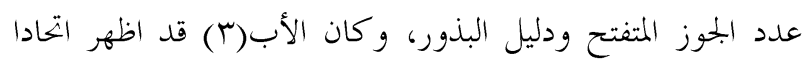

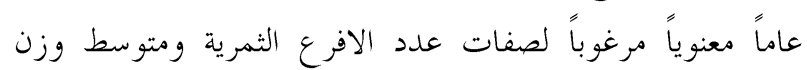

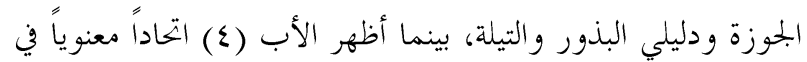

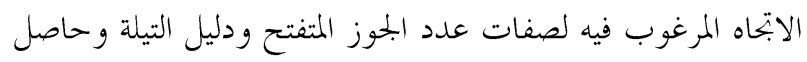

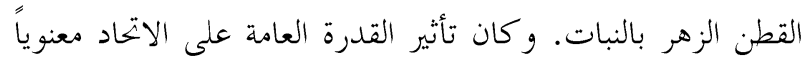
مرغوباً في الآب (0) لصفات عدد الجوز المتفتح ودليل البذور وحاصل القطن الزهر بالنبات وفي الأب (7) لصفة متوسط وزن الته الجوزة فقطـ في حين لم يبدي الأب (V) تأثيرات معنوية لأية صفة.

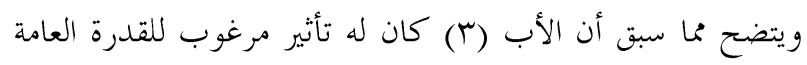

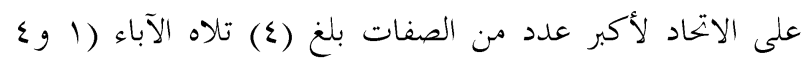
و (T) فالأب (r)، ويستنتج إمكانية الاستفادة منها في برامج التربية لتحسين الصفات التي تميزت بها، وقد حصل باحثين آخرين من

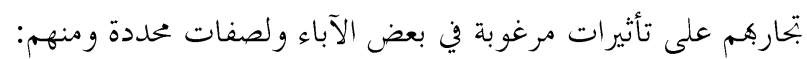
Azhar

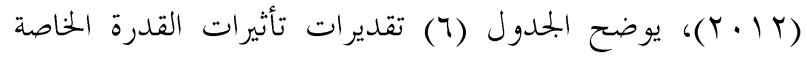
على الاتحاد لجميع الهجن وللصفات جميعها، ويلاحظ لصفة ارتفاع

جدول ه. تقديرات تأثير القدرة العامة في الاتحاد للأصناف الأبوية وللصفات المدروسة

\begin{tabular}{|c|c|c|c|c|c|c|c|}
\hline \multicolumn{7}{|c|}{ الصفات } & \multirow[b]{2}{*}{ الاصناف } \\
\hline حاصل القطن الزهر & دليل التيلة & دليل البذور & $\begin{array}{c}\text { وزن الجموزة } \\
\text { وغم) }\end{array}$ & عدالنبات الجوز & الثمرية بالنبات & $\begin{array}{c}\text { ارتفاع النبات } \\
\text { (سم) }\end{array}$ & \\
\hline$\cdot, \neg \vee 9-$ &., $011-$ & $\cdot, \wedge \circ Y-$ & 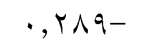 & $1, v \leq q$ & $\cdot, r \varepsilon \varepsilon$ & $\neg, Y \circ \wedge$ & 1 \\
\hline., $791-$ & $\cdot, .74$ & $\cdot, Y \wedge 0$ & $\cdot,, \leqslant \wedge-$ & דוז, & $\cdot, r \cdot 1-$ & $V, V \circ V-$ & $r$ \\
\hline $1 \cdot, 7 \backslash \wedge-$ &., 0.1 &., $0 \wedge 1$ & $\cdot, r q \mu$ & $r, r \cdot r_{-}$ & $\cdot, \mu \varepsilon \varepsilon$ &., $019-$ & $r$ \\
\hline 9,071 & $\cdot, 110$ &., $109-$ & $\cdot, \cdot V \varepsilon-$ & $r, \Sigma V T$ & $\cdot, \cdot 79$ & •,人从ו- & $\varepsilon$ \\
\hline$\Lambda, q \mu$ & $\cdot,+r \Lambda$ & $\cdot,\{\wedge q$ & $\cdot, \cdot \leq \varepsilon$ & $1, Y \backslash V$ & $\cdot, \cdot, r \cdot-$ & $\cdot, 9 \vee V$ & 0 \\
\hline$\varepsilon, \cdot Y r_{-}$ & $\cdot, .0 \leqslant-$ & $\cdot, \cdot \vee \vee \vee-$ & $\cdot, I Y Y$ & $1, \varepsilon \wedge r_{-}$ & . & $1, \pi r r$ & 7 \\
\hline$r,\{q 1-$ & $\cdot, Y \cdot \Lambda-$ & $\cdot, Y \backslash V-$ & $\cdot, \cdot \sum \wedge-$ & $1, \cdot \vee 9-$ & $\cdot, 1 \cdot v$ &., 049 & V \\
\hline $1, \pi 17$ & $\cdot, 1, \varepsilon$ & $\cdot, \mid 1+1$ & $\cdot, \cdot, 11$ & $\cdot, r \cdot q$ & $\cdot, \pi r V$ & $1, \pi{ }_{1}$ & $\mathrm{SE}\left(\mathrm{g}_{\mathrm{i}}\right)$ \\
\hline
\end{tabular}


جدول ا . تقديرات تأثير القدرة الحاصة على الحلط لكل هجين من هجن الجيل الثاين للصفات المدروسة الصفات

\begin{tabular}{|c|c|c|c|c|c|c|c|}
\hline 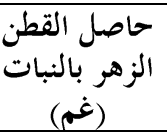 & دليل التيلة & دليل البذور & وزن الجوزة & عالنبات الجوز & الثمرية بالنبات & ارتفاع النبات & الهجن \\
\hline $1 \varepsilon, \Sigma Y Y$ &., $770-$ & $r, \ldots \Lambda-$ & $., 0,0-$ & $0,1 Y V$ & $\cdot, \varepsilon \vee \vee$ & $r, \cdot \leq \varepsilon-$ & $r \times 1$ \\
\hline $0,171-$ & $\cdot, r \vee q$ & $1, \pi 4 r$ & $\cdot, Y 00$ & $r, 79 \wedge-$ & $1,771-$ & $0, Y \wedge 1-$ & $r \times 1$ \\
\hline$\cdot, 09 \pi$ &., $0 . \leqslant-$ & $\cdot, \vee \neg\{\varepsilon-$ & $\cdot, \cdot \vee \vee q-$ &., 97 & $\cdot, \cdot 7 \cdot-$ & $11, \pi 4 \pi$ & $\varepsilon \times 1$ \\
\hline$r, \Gamma \leq \tau$ & $\cdot, \vee \leqslant \wedge-$ & $1,7 \vee 9-$ &., $.9 V-$ & $1,79 \pi$ & $1,7 r V-$ &., $111-$ & $0 \times 1$ \\
\hline $1 \varepsilon, Y q \vee-$ & $\cdot, \pi r \varepsilon$ & $\cdot, \nvdash \vee q-$ & $\cdot, \cdot r_{0}$ & $r, T \vee \varepsilon-$ & $1, \cdot r \mu_{-}$ & $1,\{7 V-$ & $7 \times 1$ \\
\hline$\Lambda, \vee \cdot \vee-$ & $1, r \leqslant 7$ & $r, Y \cdot T$ & . & $r, r \vee \tau_{-}$ & $1, r r_{0}$ & $7, V T r-$ & $v \times 1$ \\
\hline$\Lambda, V Y r_{-}$ & $\cdot, \cdot T r$ & $\cdot, \varepsilon \cdot \wedge-$ &., $1 \wedge 7_{-}$ & $r, \sum 70-$ & T,OYT- & $r, \uparrow \ldots-$ & $r \times r$ \\
\hline$r, \Upsilon \wedge 0_{-}$ & $\cdot, V \backslash r$ & $\cdot, r \neg \wedge-$ & $\cdot, Y) \varepsilon$ & $\cdot, \wedge \uparrow \vee-$ & $\cdot, 7 \wedge \leqslant$ & r,OVA & $\varepsilon \times Y$ \\
\hline $0, v 01$ & $\cdot, \vee>9$ & 1,911 &., 490 & $\cdot, \vee \backslash \backslash \wedge-$ & $\cdot, \cdot, \leqslant 9-$ & r,ov. & $O X Y$ \\
\hline $11, \cdot 29$ &., 001 & $\cdot, \mu_{0}$ &., 101 & $r, r \leqslant q$ &., 700 & $|r, \wedge \wedge|$ & $T \times Y$ \\
\hline$\varepsilon, T V Y-$ & $1, Y \circ \Lambda-$ & $\cdot, Y \cdot O-$ &., $191-$ & $1, \cdots r$ & $\cdot, T \cdot r$ & $0, r \circ Y_{-}$ & $V \times Y$ \\
\hline 1,704 & .9 $95 \leqslant-$ & $\cdot, \wedge \uparrow \varepsilon-$ & $\cdot, \cdot V \mu$ & $\cdot, \wedge \wedge \wedge$ & $\cdot, 119$ & $r, \Lambda \cdot V$ & $\varepsilon \times r$ \\
\hline $1 r, 7 \leqslant q-$ & $\cdot, 19 \cdot-$ & $1, r \leqslant 0-$ & $\cdot, r \backslash Y-$ & $r, .0 Y_{-}$ & $\cdot, \mathrm{V} \cdot \mathrm{Y}$ & ג & $0 \times r$ \\
\hline$r, \wedge \wedge r$ & $\cdot, r \wedge 0-$ & $\cdot, 7 \vee q-$ & $\cdot, \cdot \wedge 9-$ & $1, \vee \vee T$ & ., YYK- & $9,7 \leq \leqslant$ & $7 \times r$ \\
\hline $19, .71$ & $\cdot, \Lambda T r$ & $1, I V Y$ & $\cdot, r \mid r_{-}$ & $0, V Y I$ & $r, \varepsilon \mu_{0}$ & $V, V R r-$ & $V \times r$ \\
\hline Q,RYY &., $0 \wedge \varepsilon-$ & $r, \xi \uparrow \wedge-$ & $\cdot, 0 \wedge \wedge$ & $r, \Sigma q \wedge-$ & $\cdot, \wedge \wedge \nearrow_{-}$ & $0, \cdot Y Y-$ & $0 \times \varepsilon$ \\
\hline$\Lambda, 07 \pi$ & $\cdot, 991-$ & $1,049-$ & .,OYK- & T,HYT & $\cdot, Y 01$ &., 907 & $7 \times \varepsilon$ \\
\hline$r, 009-$ & $1, \varepsilon 90$ & $r, 190$ &., $11 \%-$ & $\cdot, r \cdot \wedge$ &., $.0 \mathrm{~V}$ & $\varepsilon, \varepsilon 7 V-$ & $v \times \varepsilon$ \\
\hline.,$\cdot Y Y$ & $\cdot, \cdot v_{0}$ & $\cdot, Y \wedge \vee-$ & $\cdot, \cdots \wedge-$ & $1, \cdot 1 \leqslant$ & $\cdot, Y \wedge \varepsilon$ & $\cdot, \Lambda \circ Y_{-}$ & $7 \times 0$ \\
\hline r,Tro & $\cdot, \ldots$ & r,o0. & $\cdot, \varepsilon \cdot Y-$ & $r, \Sigma V_{0}$ & $1,10 \mathrm{~V}$ & r, \&YY- & $V \times 0$ \\
\hline $17, \wedge \uparrow \wedge-$ & $\cdot,|Y|$ &., 911 &., .91 & $r, 0.1-$ & $\cdot, 791-$ & $18,907-$ & $V \times y$ \\
\hline$r, \wedge \uparrow \wedge$ & $\cdot r \cdot r$ & $\cdot, r \wedge r$ & . & $\cdot, \wedge १ \wedge$ & $\cdot, 9 \wedge 1$ & 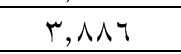 & $\mathrm{SE}\left(\mathrm{S}_{\mathrm{ij}}\right)$ \\
\hline
\end{tabular}

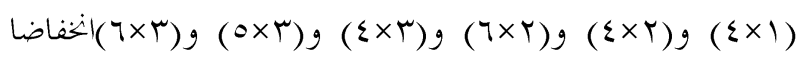

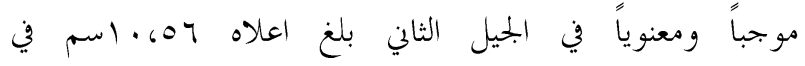
الهجين(س T/)، في حين حصلت زيادة معنوية في الجليل الثاني في

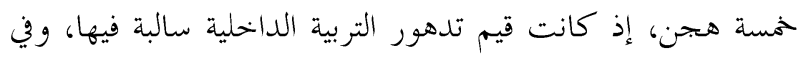
صفة عدد الأفرع الثمرية كانت قيم قيم تدهور التربية الداخلية للهجن(XXY) و(

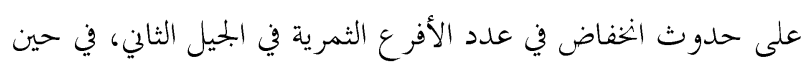
أظهرت ثلاثة هجن زيادة في عدد الأفرع الثمرية في الجيل الثاني.

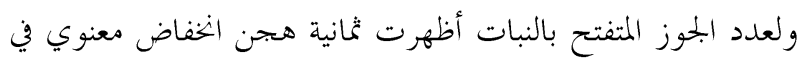

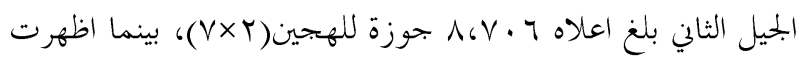

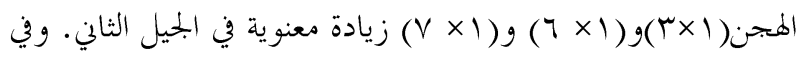

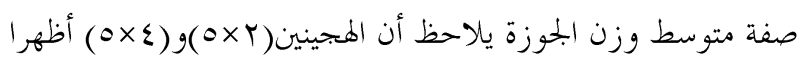

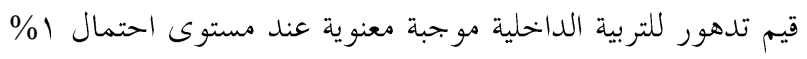
دلالة على انخفاض مهم في متوسط وزن الجوزة في الجيل الثاني لهما،
وعند ملاحظة تأثيرات القدرة العامة على الاتحاد للآباء التي

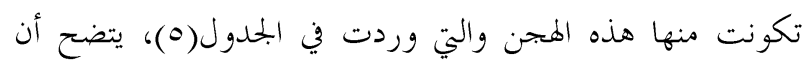

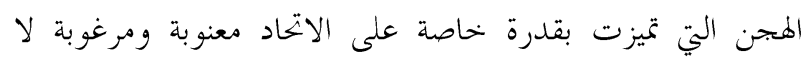
يشترط في أبويها أن يكونا ذوي تأثيرات عامة على الاتحاد عالية، وي الوقت نفسه ليس من الضروري أن ينتج الأبوان اللذان لما

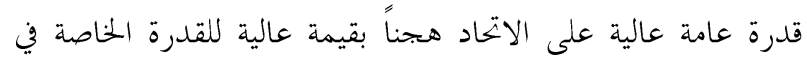

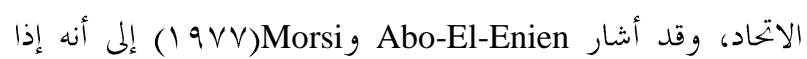
كان لهجين معين قيمة عالية لتأثير القدرة الخاصة على الاتحاد و كان

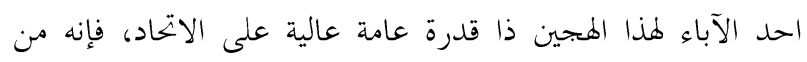
الممكن استغلال هذا الهجين لتحسين تلك الصفات. يبين الجمدول

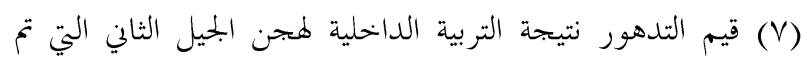

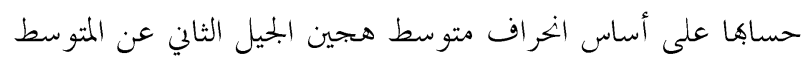
المتوقع لهجين الجيل الأول، و كانت قيم تدهور التربية الداخلية للصفات المدروسة كما يأتي: في صفة ارتفاع النبات أعطت الهجن الهجن 
للصفات تعزى إلى ظاهرة التباين الفائق الحدود في الجيل الثاني (Eissa) الثاني(Hassan، 99 V I ) والى التأثيرات الجينية غير الإضافية الموروثة

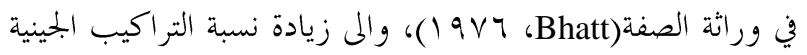
المتشابهة اليت تؤثر على الصفة في الجيل الثاني الذي يغير من متوسط

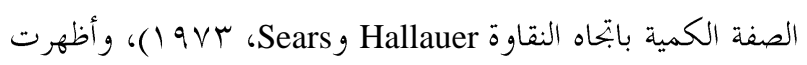
بعض الهجن قيماً موجبة ومعنوية لتدهور التربية الداخلية للصفات النفاو

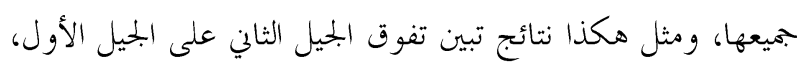
وقد عزا Hassan 99 V) (19) سبب ذلك إلى التناقص الكبير في الأليالت السائدة المؤثرة على الصفة في التركيب الوراثي للآباء، وكذلك إلى ظاهرة الانعزال المتجاوز الحلدود في الجيل الثاني. وقد حدد الصفار(1 . . r) في دراسة أن التربية الداخلية لكي يكون لها

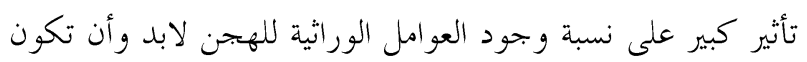
مصحوبة بنوع من الانتخاب،
في حين أظهرت عشرة هجن قيم تدهور للتربية الداخلية سالبة ووعنوية، أي أن متوسط وزن الجوزة كان أفضل في الجيل الثاني. ولصفة دليل البذور كانت قيم تدهور التربية الداخلية موجبة

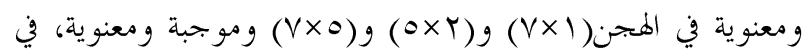

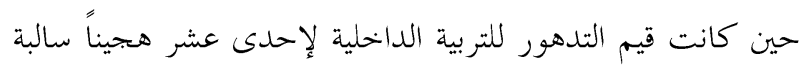
وومنوية. ولصفة دليل التيلة ظهرت قيم تدهور التربية الداخلية موجبة و معنوية في الهجن (VX) بينما كانت سالبة ومعنوية في تسعة هجن أخرى، وأخيراً لصفة حاصل القطن الزهر بالنبات كانت قيم تدهور التربية الداخلية لثمانية هجن موجبة ومعنوية، وبلغ أعلى الخفاض في الجيل الثاني

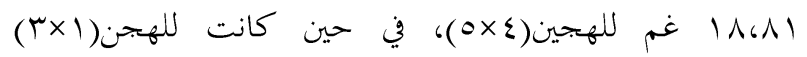

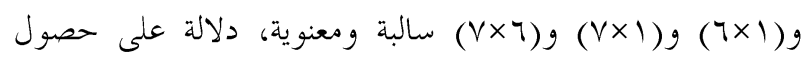
زيادة معنوية فيها في الجيل الثاني بلغت بr, r.,

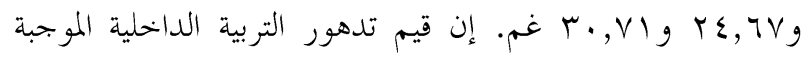

الجدول V. قيم التدهور نتيجة التربية الداخلية لهجن الجيل الثابي للصفات المدروسة

\begin{tabular}{|c|c|c|c|c|c|c|c|}
\hline \multicolumn{7}{|c|}{ الصفات } & \multirow[b]{2}{*}{ الهجن } \\
\hline الز هر بالنبات القطن & دليل التيلة & دليل البذور & وزن الجوزة & عدالنبات الجوز & الثمرية بالنبات & ارتفاع النبات & \\
\hline *I $1 \varepsilon, \circ \wedge \mu$ & $\cdot, \circ<\varepsilon-$ & 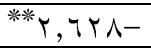 & ***, , VYY- & 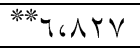 & $\cdot, \boldsymbol{\mu} \cdot-$ & $* 0,711-$ & $r \times 1$ \\
\hline *IT,YYY- & $\cdot, \cdot 11$ & • & $\cdot, \cdot \varepsilon \varepsilon-$ & *, . 10- & ***十,人リ1- & $*_{\Upsilon}, \Upsilon \uparrow V-$ & $r \times 1$ \\
\hline$\cdot, r \cdot \varepsilon-$ & ***, , $\wedge \vee \varepsilon-$ & ${ }^{* * *} Y, \cdot V Y-$ & $\cdot, r \neg V-$ & $1, Y 17$ & $\cdot, 7 \ldots-$ & ***1.,111 & $\varepsilon \times 1$ \\
\hline$\cdot, \cdot \wedge \wedge-$ & **** $1, .07-$ & ****T,YYY- & $\cdot, \cdot \wedge 9-$ & $r, \Sigma \leqslant 0$ & **** Y, \TV- & $0,111-$ & $0 \times 1$ \\
\hline *** Y Y $6.0-$ & $\cdot, \cdot \Lambda \mu$ & $* 1,1 \leq 1-$ & ***, , $\leqslant 0 .-$ & 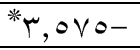 & ******, $1, \vee \wedge 9-$ & $* 4,111-$ & $7 \times 1$ \\
\hline ***T $Y \xi, T \vee-$ & 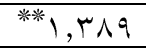 & $* 1$, rVA & $\cdot, 17 \mathrm{~V}-$ & 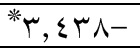 & *** Y,00 Y- & **** $Y \backslash, \mid V-$ & $v \times 1$ \\
\hline$r, q \cdots-$ &., .11 & $\cdot, \wedge \uparrow q-$ & ****, , โ\V- & $1,1 \leq \varepsilon-$ & 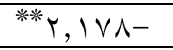 & $.64 \wedge 9$ & $r \times r$ \\
\hline 9,1990 & $*, 009$ & 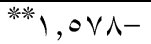 & $\cdot, \cdot \leq \varepsilon$ & $r, 107$ & ***1,,$\ldots$ & $* 0, V \ldots$ & $\varepsilon \times Y$ \\
\hline$* 17, Y, 1$ & *., $\uparrow \vee \wedge$ & $*$ I, rVY & ****, IYY & Y,TVY & $\cdot, \Sigma \ldots$ & $r, q \leq \varepsilon$ & OXY \\
\hline$* 17,179$ &., $01 \mathrm{~V}$ & $\cdot, \Sigma \backslash \mu-$ & 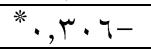 & **** $\varepsilon, 9 \wedge \uparrow$ & **** $1, \Gamma \vee \wedge$ & **** $|Y, T|$ & $7 \times Y$ \\
\hline **** $\mid \vee,\rceil \mid$ & *. $7 \vee \backslash-$ &., $09 \leq-$ & ***, VYY- & ${ }^{*} \wedge, \vee \vee \neg$ & $\cdot, \wedge 11$ & $r, 111-$ & $V \times Y$ \\
\hline 7,017 & 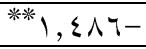 & ****, १人ץ_ & $\cdot, \mid \backslash \vee \wedge-$ & $1, \wedge T \varepsilon$ & $\cdot, 7 \wedge 9$ & ${ }^{*} \wedge, 111$ & $\varepsilon \times \mu$ \\
\hline $11, \Sigma Y Y-$ & *., $79 .-$ & 档।, V...- & $\cdot, Y \backslash \vee-$ & $\cdot, T \wedge \mu_{-}$ & $\cdot, \vee \wedge 9$ & **** $\wedge, \wedge \wedge 9$ & $0 \times r$ \\
\hline$\varepsilon, Y \backslash r-$ & 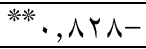 & *1,r०Y- & ***, ฯ「人ー & Y, ₹१V & سזו, & ****,, 04 & $7 \times r$ \\
\hline * $I \leq, 7 \leq Y$ &., $01 \varepsilon$ & • & 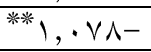 & ${ }^{* * *} \wedge, \zeta \backslash \vee$ & * & $0,17 \mathrm{~V}-$ & $V \times r$ \\
\hline 粎 $|\wedge, \wedge|$ & ***), $1 \wedge 7-$ & **** T, TVY- & ****,, I $\{\varepsilon$ & ., క०น- & - & - & $0 \times \varepsilon$ \\
\hline$\wedge, \vee Y Y$ & ***,, $0 \leqslant r$ & *** $Y, 991-$ & ***,, $90 .-$ & $*, V \backslash \varepsilon$ & $* 1,1 \vee \wedge$ & $r, \ldots$ & $7 \times \varepsilon$ \\
\hline * I ๆ, $\{9 \varepsilon$ & *., 099 &., .71 & ** & *** $\varepsilon, Y \circ 1$ & $\cdot, \wedge 11$ & $1, r \vee \wedge$ & $V \times \varepsilon$ \\
\hline Y,乏०Y- & $\cdot, \varepsilon \cdot \wedge-$ & $* 1, \cdot \vee \varepsilon<-$ & ., rrq- & $*^{*}, \Lambda \cdot r$ & $\cdot, \vee \leqslant \varepsilon$ & $r, 007-$ & $7 \times 0$ \\
\hline * $1 \varepsilon, 199$ & *., $799-$ & 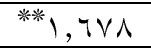 & **,rYY- & *** $\vee$ マ $\wedge$. & $\cdot, \wedge \leq \varepsilon$ & ${ }^{*} \wedge, 1 \leq \varepsilon-$ & $V \times 0$ \\
\hline ****, vi- &., $1 \circ \mathrm{V}-$ & $\cdot, 0 \cdot 1-$ & ***, , 91V- & $\cdot, \circ \vee \cdot-$ & *1, , V^- & 粎 $1,71-$ & $V \times 7$ \\
\hline
\end{tabular}


جدول ^. مكونات التباين المظهري وبعض المعالم الوراثية للصفات المدروسة

\begin{tabular}{|c|c|c|c|c|c|c|c|}
\hline 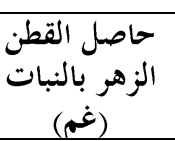 & دليل التيلة & دليل البذور & وزن الجمزة & عالنبات الجوز & عدالثمرية الافرع & $\begin{array}{c}\text { ارتفاع النبات } \\
\text { السم) }\end{array}$ & والتباين \\
\hline$\wedge \wedge, \circ 9 \wedge$ &., 109 & $\cdot, \varepsilon \cdot q$ & $\cdot, \cdot \leq 0$ & $v, 11 \varepsilon$ & & $T V, r \leqslant T$ & \\
\hline \pm & \pm & \pm & \pm & \pm & --- & \pm & $\sigma^{2} \mathrm{~A}$ \\
\hline YO,TYE &., .01 & $\cdot, 1 Y \varepsilon$ &., .17 & $r, \cdot r r$ & & $\wedge, \neg \backslash \wedge$ & \\
\hline $\mid \Gamma \varepsilon, \cdot r \varepsilon$ & $\cdot, r \wedge r$ & $1,9 \cdot r$ & $\cdot, \cdot \varepsilon q$ & $1 \cdot, \cdot 19$ & $\cdot, r q V$ & $\{r, \neg\}\rceil$ & \\
\hline \pm & \pm & \pm & \pm & \pm & \pm & \pm & $\sigma^{2} \mathrm{D}$ \\
\hline$\varepsilon 0, \cdot r \mu$ & $\cdot, 1 \leqslant \Lambda$ &., 717 & $.6 . r V$ & $r, r>q$ & $\cdot,\{9 \leqslant$ & $1 \Lambda, V r r$ & \\
\hline $0 \xi, O V Y$ & $\cdot, r \leqslant r$ &., $0 \leqslant r$ & $\cdot, r \cdot \Lambda$ & $r, \ldots r$ & $r, 0 \wedge 0$ & OT,YOY & \\
\hline \pm & \pm & \pm & \pm & \pm & \pm & \pm & $\sigma^{2} \mathrm{E}$ \\
\hline $1 \cdot, \pi \mid r$ &., .70 & r., & ., &., 071 & $\cdot, 7 \vee \wedge$ & $1 ., 7 \pi 1$ & \\
\hline $1, V \mu q$ & $r, 197$ & $r, \cdot \varepsilon \wedge$ & $1, \Sigma V Y$ & $1,7 \wedge r$ & --- & $1, \vee \wedge 9$ & $\overline{\mathrm{a}}$ \\
\hline., 4197 &., $1 \vee 97$ & $\cdot, 1 \leqslant r_{0}$ & $\cdot, 1 \leqslant \wedge 9$ & $\cdot, \mu \circ Y \varepsilon$ & --- & $\cdot, r \backslash \leqslant r$ & $\mathrm{~h}_{\mathrm{ns}}^{2}$ \\
\hline$\cdot, \Lambda \cdot \mu$ & $\cdot, 71 Y V$ & $\cdot, 1 \cdot 99$ & $\cdot, r \mid \cdot r$ & $\cdot, \wedge 01 Y$ & $\cdot, \cdot \vee \vee \neg \varepsilon$ &., $00 V_{0}$ & $\mathrm{~h}_{\mathrm{bs}}^{2}$ \\
\hline 9,470 & $\cdot, Y q V$ & $\cdot, \sum Y V$ & $\cdot, 1 \leq \varepsilon$ & r, $\vee \wedge q$ & --- & $\varepsilon, Y \circ T$ & GA \\
\hline $1 Y, 997$ & $0, \varepsilon Y q$ & $\varepsilon, Y Y I$ & $r, 19 \varepsilon$ & $17, Y Y V$ & --- & $r, 0 T V$ & GA\% \\
\hline
\end{tabular}

هذه الصفات الأخيرة عن طريق الانتخاب الإجمالي، اما التحسين

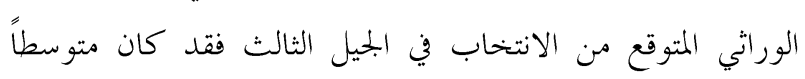
لصفتي عدد الجوز بالنبات وحاصل القطن الزهر، حيث بلغ فيهما

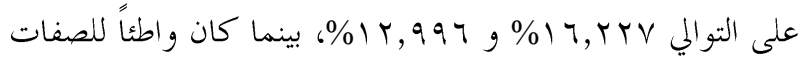

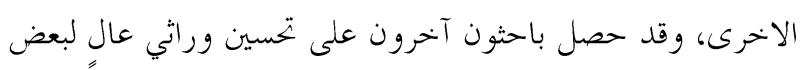

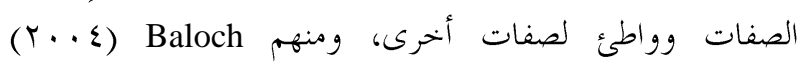

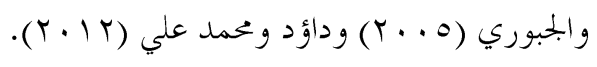

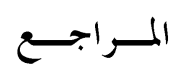

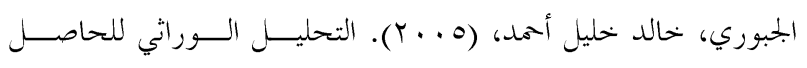

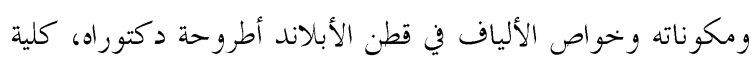

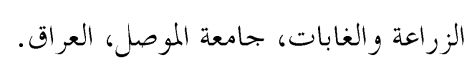

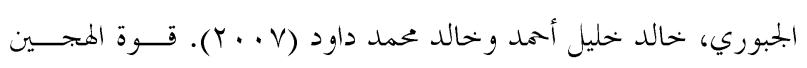

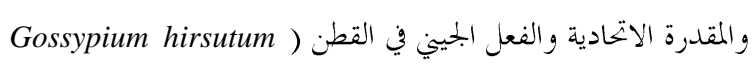
L.

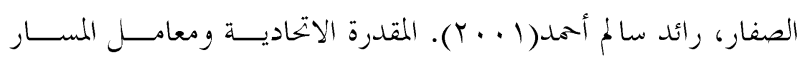

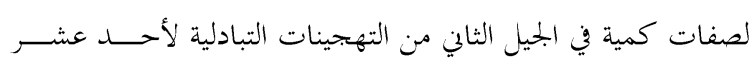

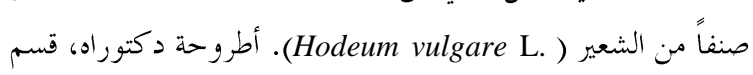
علوم الحياة، كلية العلوم، جامعة الموصل.

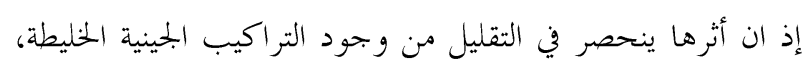

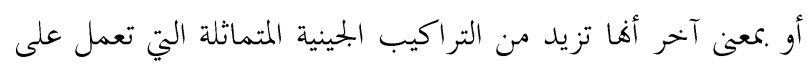

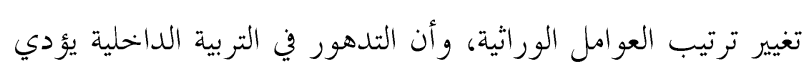

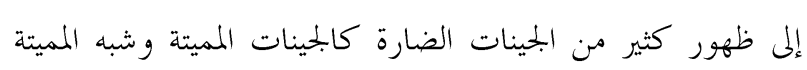

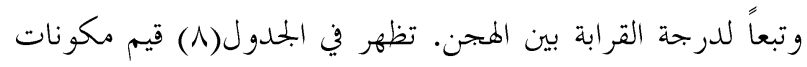

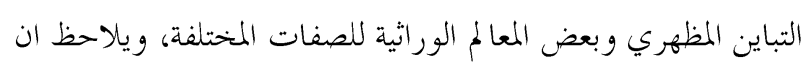

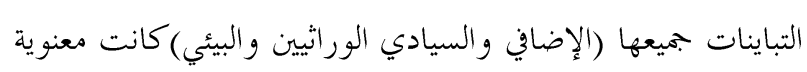

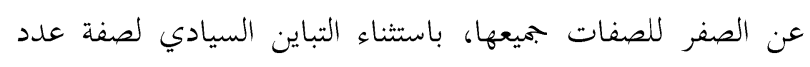

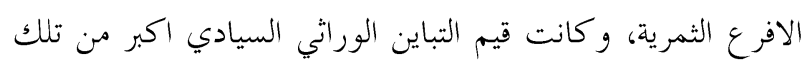

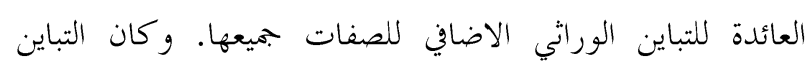

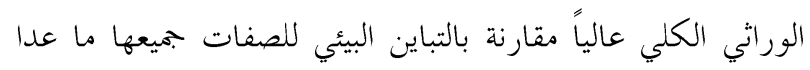

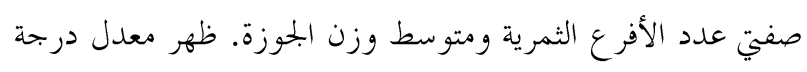

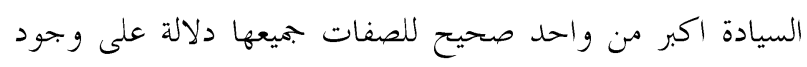

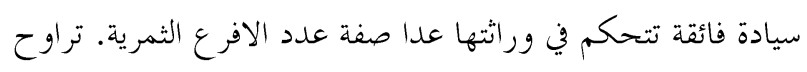

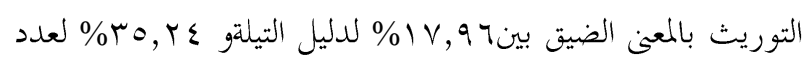

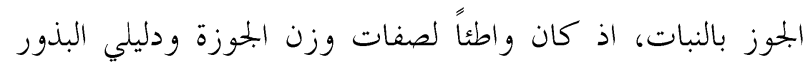

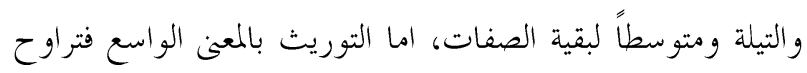

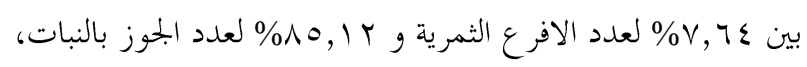
اذ كان واطئاً لصفتي عدد الافرع الثمرية ووزن الجوزة لأو ومتوسطاً

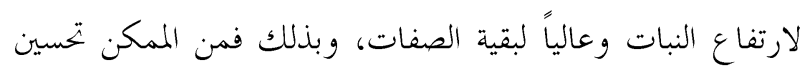


Griffing, B. (1956). Concept of general and specific combining ability in relation to diallel crossing systems, Aus. J. Biol. Sci. 9:463-493.

Hassan, E. E. (1997). Combining ability and factor analysis in durum wheat (Triticum turgidum). Zagazig J. Agric. Res. 24(1):23-36.

Hallauer, A. R. and J. H .Sears (1973). Changes in quantitative traits associated with inbreeding in a synthetic variety of maize. Crop. Sci. 13:327-333.

Iqbal, M., K. Hayat, M. Atiq and N. I. Khan (2008). Evaluation and prospects of F2 genotypes of cotton (Gossypium hirsutum) for yield and yield components. Int. J. Agri. Biol., 10(4): 442-446.

Iqbal, M., M. Z. Iqbal, M. A. Chang, and K. Hayat. (2003). Yield and fiber quality potential for second generation cotton hybrids. Pakistan Journal. Boil. Sci. (Pakistan). 6(22):1883-1887.

Kempthorne, O. (1957). An introduction to genetic statistics. Jihn Wiley and sons, New York, U S A.

Khan, N. U. (2003). Genetic Analysis, Combining Ability and Heterotic Studies For Yield, Its Components, Fiber and Oil Quality Traits In Upland Cotton(G. hirsutum L.), Ph.D. Dissertation, Sindh Agric. Univ. Tandojam, Pakistan.

Khan, N. U., G. Hassan, M. B. Kumbhar, K. B. Marwat, M. A. Khan, A. Parveen, U. Aiman and M. Saeed (2009a). Combining ability analysis to identify suitable parents for heterosis in seed cotton yield, its components and lint $\%$ in upland cotton, Ind, Crops Prod., 29: 108-115.

Khan, N. U., G. Hassan, K. B. Marwat, Farhatullahi, M. B. Kumbhar, A. Parveeni, Umm-E- Aimani, M. Z. Khan and Z. A. Soomro (2009b). Diallel analysis of some quantitative traits in Gossypium hirsutum L, Pak, J, Bot, 41(6): 3009-3019.

Makhdoom, K. (2011). Combining Ability Estimates Through Line $\mathrm{x}$ Tester Analysis and Heritability In Upland Cotton, M. Sc. (Hons,) Thesis, Khyber Pakhtunkhwa Agric. Univ. Peshawar, Pakistan.

Mather, K., and J. L. Jinks (1982). Introduction to biometrical genetics. Chapman and Hall ltd, London.

Singh, R. K. and B. D. Chaudhary (2007). Biometrical methods in quantitative genetic analysis. Kalyani Publishers, New Delhi, 304p.

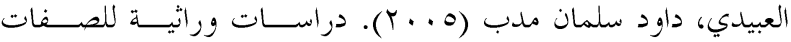

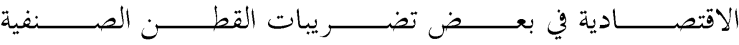

$$
\begin{aligned}
& \text { (Gossypium hirsutum L.) }
\end{aligned}
$$

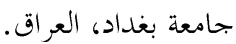

$$
\begin{aligned}
& \text { العذاري، عدنان حسن محمد (999 199). أساســيات في الوراثــة. وزارة } \\
& \text { التعليم العالي والبحث العلمي. جامعة الموصل. } \\
& \text { داؤد، خالد محمد واكرم عبد الرزاق محمد علي (r ( + (T). تحليل التهجين } \\
& \text { التبادلي في الجليل الثاني لصفات الحاصل و بعض مكوناتــهـ في قطــن } \\
& \text { الابلند (ossypium hirsutum L.). بحلة زراعة الر افدين } \\
& \text { علي، عبده الكامل عبد الله (999 199)، الغزارة الهجينية والفعل الجــيني في }
\end{aligned}
$$

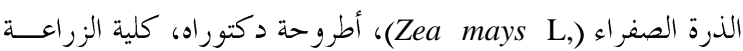

$$
\begin{aligned}
& \text { والغابات، جامعة الموصل، العراق، }
\end{aligned}
$$

Abo-El-Enein, R. A. and L. R. Morsi (1977). Heterosis and combining ability in barley by diallel analysis. Egypt. J. Genet. Cytol. 6: 84-97.

Agarwal, V. and Z. Ahmad (1982). Heritability and genetic advance in triticale. Indian J. Agric. Res. 16:19-23.

Allard, R. W. (1960). Principles of plant breeding. John Wiley and Sons. Inc. New York.

London pp: 485.

Azhar, F. M., M. A. Khan, and N. Mahmood. (2004). Combining ability analysis of fiber characteristics in Gossypium hirsutum L. International Journal. Agric. and Biology (Pakistan). 6(2): 272-274.

Baloch, M. J. (2004). Genetic variability and heritability estimates of some polygenic traits

in upland cotton. Pakistan. J. of Sci. 47(6):451-454.

Basal, H., and I. Turgut (2003). Heterosis and combining ability for yield components and fiber quality parameters in a half diallel cotton ( $G$. hirsutum L.) population, Turk. J. Agric. For. 27:207-212.

Bhatt, G. M. (1976). Variation of harvest index in several wheat crosses. Euphytica 25: 41-50.

Eissa, M. M. (1993). Combining ability for main spike characteristics in durum wheat (Triticum turgidum var durum). Zagazig J. Agric. Res. 20:1673-1681.

Gomma, M. A. M., and A. M. A. Shaheen (1995). Heterosis, inbreeding depression, heritability and type of gene action in two intra-barbadense cotton crosses. Annals Agric. Sci. Ainshams Univ. 40(1):165-176. 


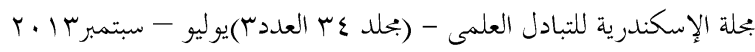

\title{
SUMMARY \\ Combining Ability of Upland Cotton Genotypes over F2 Generation Of 7x7 Diallel Cross
}

\begin{abstract}
Kh. Kh. A-Juboori
Cotton varieties, Coker30, SP8886, Lachata, Montana, Halab33, AC22 and Gourd26, and all their $F_{2}$ half diallel crosses were planted at Al-Hawija, Kirkuk Governarate in $1^{\text {st }}$ of May, 2012 using randomized complete block design with three replications to study general and specific combining abilities for parent and crosses respectively and estimation of phenotypic variance components and some genetic parameters for characters: plant height, number of fruiting branches per plant, number of bolls per plant, boll weight, seed index, lint index and seed cotton yield per plant. results obtained from analysis of variance showed that mean square of genotypes, general and specific combining ability was proven to be significant for all characters except that of genotypes and general combining ability for number of fruiting branches. The variety Lachata appeared as a good general combiner, and (SP8886 x Halab33), (Lachata x Gourd26) crosses as good specific combiners for larger number of characters. The variances (additive, dominance and environmental)

dominance one for number of fruiting branches, and the dominance variance was higher than additive one for all characters. The total genetic variance was high as compared with environmental variance for all characters except number of fruiting branches and boll weight. The average degree of dominance was higher than one for all characters indicating the presence of over dominance with exception of number of fruiting branches. Narrow sense heritability ranged from $17.96 \%$ for lint index to 35.24 for boll number, as was low for boll weight, seed index and lint index and moderate for other characters, while broad sense heritability ranged from $7.64 \%$ for number of fruiting branches and $85.12 \%$ for boll number, as was low for number of fruiting branches and boll weight, moderate for plant height and high for other characters. Genetic advance as percent from character mean in the third generation was moderate for boll number and seed cotton yield where reaches in them $16.227 \%$ and 12.996 respectively, while it was found to be low for other characters.
\end{abstract} appeared significant from zero for all characters, except 